\title{
CONSERVATIVE TREATMENT FOR ANAL FISSURE - FOR HOW LONG? - REVIEW OF THE LITERATURE AND PERSONAL EXPERIENCE
}

\author{
Valeriu Șurlin $^{1,2}$, Sorin Scurtu ${ }^{2}$ \\ ${ }^{1} 1$ st Clinic of Surgery, Clinical County Emergency Hospital of Craiova, Craiova, Romania \\ ${ }^{2}$ PROCTOLINE Clinic Craiova, Craiova, Romania
}

\begin{abstract}
Anal fissure $(A F)$ is a common proctologic disease and health problem, with potential for chronicity, causing the patient a debilitating suffering. The pathogenesis, in most of the cases consists in a vicious circle of pain-internal anal sphincter (IAS) spasm-decrease blood irrigation of mucosa-delay of healing. Treatment should aim to break this mechanism by cutting or relaxing the IAS and restoring adequate blood flow to promote healing. If anal stretch is becoming history, surgical sphincterotomy has the highest rate of healing and the least percentage of recurrence, but incontinence for flatus and stool may occur so "medical or "chemical" sphincterotomy using calcium channel blockers (CCB), nitric oxide donors, botulinum toxin injection are used with success rates from $50 \%$ to over $90 \%$, also in combination with other conservative measures like warm sitz baths, stool softeners, high fiber and more liquid daily ingestion. All of those therapeutic measures are also stated in current guidelines. The question we tried to answer in this paper is for how long we can prolong the conservative, non-operative treatment in AF. Based upon literature research and our personal experience we may state that the earlier institution of medical specific treatment (CCB, nitric oxide donors, botulinum toxin) has more chances to heal the patient and avoid surgery. Success is also dependent on the good communication with the patient, detailed explanation of the purpose of the treatment, how to assess himself the evolution, and employ botulinum toxin earlier if patient is less compliant to topic treatment. Conservative non-operative management should be pushed as long as it is correctly applied and progression is made both from subjective and objective point of view and patient is compliant and content.
\end{abstract}

Key words: anal fissure, conservative treatment, internal anal sphincter, chronic anal fissure.

\section{Introduction}

The definition of an anal fissure according to the Manual of Coloproctology, second edition is a painful tear in the epithelial lining of anoderma anterior or posterior, distal to the dentate line [1].

The etiology is considered to be multifactorial, including passage of hard stools, forceful and bulky defecation, diarrhea, trauma and other causes.

From the objective point of view, we encounter 2 types of anal fissures in our practice, as every other proctologist does. The recent fissures, usually less than 2 weeks, which are linear in shape, sharp, clean edges, bleeding during the examination that we may call acute anal fissures (AAF).

The other aspect is an anal fissure, triangular in shape, with apex at the dentate line, base at the anal verge, a "sentinel pile" on the outside, a hypertrophic papilla at the apex, edges may be sclerous, the base of the fissure may exhibit fibers of the internal sphincter, without any objective sign of granulation tissue. In the old books, this was the classical description of an anal fissure, nowadays we consider this a chronic fissure (CAF).

Correspondence to: Valeriu Surlin, M.D., Ph.D

Clinica I Chirurgie, etaj V, SCJU Craiova, str. Tabaci nr.1

Craiova 200642, Dolj, Romania

Phone: +40740182346

E-mail: vsurlin@gmail.com

Received May $7^{\text {th }}, 2019$

\section{Pathogenesis of the Chronic Anal Fissure}

The pathogenesis of the CAF is surely a complex one, there is no single mechanism involved.

The most consistent findings in anal fissures are the hypertonicity of the internal sphincter with an elevated resting tone. This is an objective sign that we note immediately when we try to examine the patient by digital tact, that is mostly impossible, requiring sedation, local anesthesia by topic of injection, loco-regional or general anesthesia to complete the proctologic examination. Manometric studies of the resting anal tone support this objective finding [2].

Internal sphincter spasm is generated by the presence of the fissure, deep enough to expose the sensitive nerve endings and it is reflex and it is decreasing the blood flow that crosses it to reach anoderma, contributing to a delayed or no healing progression. This has been demonstrated in several studies assessing the blood perfusion using Doppler laser flowmetry [3, 4].

In most of the cases this chronic aspects of $\mathrm{AF}$ are located at the posterior and anterior commissures, only $10 \%$ of the patients having fissures at the anterior midline and in $1 \%$ both commissures being involved [5]. Anterior location is more frequent in women [6]. There is a postulate that fissures occurring on other places than anterior and posterior commissures should raise the suspicion of other diseases like Crohn disease, sexually 
transmitted disease or even AIDS [7]. Explanation of this predilection may come in the majority of cases, from the anatomy, blood supply coming from inferior rectal arteries, on both sides, crossing the internal anal sphincter to reach anal anoderma, do not have any or minimal contact in the posterior commissure in $85 \%$ of cases. This fact was demonstrated by Klosterhafen et al in. a post-mortem angiographic study [8].

A personal observation is that cicatrization may be delayed also in patients suffering from chronic diseases as diabetes mellitus or renal insufficiency, due to the alteration of small blood vessels as a result of a systemic disease. All of those features suggest that the anal fissure behaves like a chronic ischemic wound, due to a vicious circle of "pain - IAS spasm - decreased blood flow - no healing - pain". Some studies found consisting evidence for small blood vessels alterations similar to a vasculopathy. Maria et al. identified in the peripheral blood of patients with chronic anal fissures circulating antibodies against intimal endothelial cells and deposits of antigen-antibody complexes in the small branches of the rectal arteries [9].

\section{Treatment}

Not all acute anal fissures go into this vicious circle leading to a non-healing, ischemic wound. Normally, a wound in this area should heal spontaneously like any other wound in the human body, in a period of maximum 2 weeks. A part of them may indeed do so, as in the anamnesis of the patients, they report episodes of constipation, with pain after one strenuous, difficult defecation, after an episode of constipation, bleeding on paper, pain that reappears after several stools with minor bleeding, symptoms typically related to an anal fissure, but they gradually resolve and, when seen in consultation, the examination reveals normal anoderma.

Some of the patients performed by themselves sitz baths, take stool softeners or laxatives, eat more fibers and drink more liquids to soften the stools, a fact that reduces the pain after defecation and improves symptoms. Some of them consult their pharmacist or family doctor, in the best case, and are advised to use ointments, suppositories, jelly containing substances that promote local contact anesthesia, have anti-inflammatory, siccative effect, and promote cicatrization, made either from natural plants or synthetic. These products are plenty on the market, and are advertised for hemorrhoids and/or anal fissures, consisting in fact in a purely symptomatic treatment. This treatment is considered as conservative one and is used by many specialists in proctology as a first line therapy and is also included in guidelines. Treatment of CAF anal fissure should not aim to the wound itself but to the breaking of this vicious circle by any means, by addressing the internal anal sphincter spasm and improving blood flow.

From its first description in 1829 by Recamier, we have the first therapeutic measure indicated as anal stretching, a technique that will weaken the thin internal anal sphincter by overstretch, but without any control in preventing damages to the external anal sphincter, therefore it is considered historic.

The most successful treatment of the AF was surgical sphincterotomy, initially performed in the AF's bed, cutting only through the fibers of the anal sphincter that are visible, or going up to the dentate line, excising wound edges, papilla and sentinel pile a so called fissurectomy, that will rejuvenate the wound and help healing.

This operation was successful in almost all cases but left a deformity of a keyhole appearance or a groove responsible for a period of time of minor leakage of liquid drops of stools or staining the underwear. In some patients this could be permanent, in others may be present for a determined period of time.

To avoid this, recently a lateral sphincterotomy is indicated, involving the distal part of the internal sphincter. An anterior internal sphincterotomy is prohibited by the fact that external anal sphincter is weaker in this region. The success of the surgical sphincterotomy is related to the amount of internal sphincter that is cut. A complete sphincterotomy, up to the dentate line will be mostly effective in healing almost all fissures but may increase the percentage of the incontinence symptoms. Risk of incontinence may be as high as $10 \%$, especially for flatus $[10,11]$. A postal survey of patients operated for anal fissure revealed a higher rate of incontinence: $28.7 \%$ for stool and $31.5 \%$ for gas. Risk for incontinence may increase over the years because of the weakening of the anal sphincter with age [12]. If a lateral sphincterotomy is performed, a part of $25 \%$ up to $50 \%$ of sphincter maybe cut with good results in healing and risk of incontinence [1] the recurrence maybe higher or healing incomplete in worst cases, but it can be extended by a second intervention.

Another possibility is to tailor the sphincterotomy only for the fibers of internal sphincter seen in the fissure bed. Both techniques are credited with lower rate of incontinence.

The way of doing it: open or closed sphincterotomy is a matter of choice depending upon the expertise of the proctologist, we prefer to lay it open to avoid formation of abscesses, the patient will be taught to performed regular sitz baths, perform self anal dilation with ointments that include local anesthetics and even local antibiotics, but this is low grade recommendation, based upon personal expertise and not on high grade evidence.

\section{Medical Treatment}

The necessity of avoiding those side-effects pushed specialist in seeking a "Medical Sphincterotomy" or a "reversible sphincterotomy" or a "chemical sphincterotomy" in decreasing the internal sphincter tone by oral, topic medication, or botox injections.

Calcium channel blockers (CCB) Oral Nifedipine, Nifedipine gel $0.2-0.5 \%$, Diltiazem cream $4 \%$, were employed with success in treatment of AF based on the 
effect of relaxing the smooth muscle, inducing vasodilatation and reducing anal spasm. The side effect is hypotension and headache. Topic application has more local direct effect and less systemic side-effects $[13,14]$

Nitric oxide donors. Nitric oxide is a neurotransmitter that induces smooth muscle relaxation and therefore, internal sphincter relaxation. The mostly employed are glyceryl-trinitrate (GTN) and nitro glyceryn (NTG). GTN is used in concentrations of $0.2 \%$ to $0.4 \%$. Although demonstrated effective in treating $\mathrm{AF}$ [15], contrary to what we might have expected higher concentrations do not offer higher rates of healing [16]. NTG is used in concentrations from $0.1-0.6 \%$. Healing takes place usually after 6-8 weeks but sometimes it requires 12 weeks to heal. Side-effects, more often encountered if the dosage is increased, are headaches, making a part of the patients non-compliant to the treatment. The phenomenon of tachyphilaxia is also possible making necessary to increase dosage [17].

\section{Botulinum toxin (Botox)}

The role is to induce a local paralysis of the IAS for a period of 3-4 months, that should be enough for a complete healing. The effects are reversible and so are any incontinence symptoms during the treatment. Indicated for AF is the botulinum toxin type A [18]. The dosage found to be effective is 20-25 units injected in the anal sphincter. Studies regarding the dosage showed a surprising evidence of an efficiency in healing and incontinence non influenced by the dosage, formulation and type of toxin. Also the site and number of injections per session didn't influence the healing rate [19]. Lower doses of botox reduce the risk of incontinence and recurrence in the long term. The optimum injection site could not be determined [20]. For example, in our experience, we use to inject a total amount of 25 units (toxine type A), $12.5 \%$ on each side of the CAF.

\section{Comparison between Different Treatments}

A systematic review and meta-analysis of all published treatments for anal fissure that have been studied in randomized controlled trials was performed by Nelson et al. relatively recent in 2017. The conclusions are very clear supporting calcium channel blockers over GTN, with less risk for headache as side effects, a feature important for long-term treatments. The rate of healing is lesser that of lateral surgical sphincterotomy that is credited with a rather lower rate of incontinence of 3.4-4.4\%. This is probably due to performing a distal internal sphincterotomy rather than a complete one. Open LIS and closed LIS were equal in regards of efficiency [21].

Botox injections have a risk of anal incontinence, but fewer side-effects compared to nitric oxide donors [22].

\section{The Sequence of Treatment Indicated in the Guidelines}

The 2014 American College of Gastroenterology clinical guideline on the management of benign anorectal disorders made the following recommendations for anal fissure [11]:

Acute anal fissure - Providers should use nonoperative treatments (e.g., sitz baths, psyllium fiber, and bulking agents) as the first step in therapy (strong recommendation, moderate-quality evidence)

Chronic anal fissure - Providers should treat chronic anal fissure with topical pharmacologic agents (eg, calcium channel blockers or nitrates) (strong recommendation, moderate-quality evidence)

Chronic anal fissure - Providers should refer patients who do not respond to conservative or pharmacologic treatment for local injections of botulinum toxin (strong recommendation, low-quality evidence) or internal anal sphincterotomy (strong recommendation, highquality evidence) [23].

The question to be answered is for how long should we keep the patient on medical on conservative (nonsurgical) treatment before referring him to surgery?

If it is an acute anal fissure the dietary changes, sitz baths, local symptomatic ointments heal the fissure in the majority of cases [24]. Rates of healing may be up to 50-80\%. It a fissure has a chronic aspect, the response to such dietary and habits changes is rather low. In those cases this will remain as an "adjuvant" to the medical treatment [25]. We should not prolong this kind of treatment beyond 2-3 weeks at maximum, because this simply mostly symptomatic treatment, because after this period the AF is entered probably in the vicious circle mechanism that doesn't allow further healing.

In a study performed by Sileri et al, in which the patients were submitted gradually to medical treatment first, botox injection and as a last resource IS, we may find some orientating results. Beside dietary adjustment, sitz baths, initially, the patients with CAF were submitted to NTG $0.2 \%$ twice a day, applied to the fissure and to the internal sphincter for a period of 8 weeks with a rate of healing of $54 \%$. If the patient was still symptomatic after 8 weeks, Botox injections were proposed or prolongation of treatment with NTG. After 12 weeks the rate of healing was $64 \%$ for NTG. Rate of healing after Botox was $84 \%$. Patient showing an incomplete healing were submitted to either another injection of Botox or surgical sphincterotomy. LIS had a cure rate of $98.7 \%$ with no definitive incontinence on long-term follow up [26].

Personal observation come from a series of 567 patients seen in the last 4 years in consultation in our clinic as outpatient, their observation files were retrieved and data within reexamined and analyzed. There was a slight male predominance of 307/267 (1,15 x higher). Most of the patients were in the 30-50 years of age $-252(44.44 \%), 20 \%$ under 30 years, and $35 \%$ over 50 years of age. So, nearly $80 \%$ of patients were over 30 years old. Co-morbidities of arterial hypertension were 
met in 69 patients, diabetes mellitus in 31 and irritable bowel syndrome in 56 .

Beside the basic recommendations as stool softeners, sitz baths, dietary fibers, laxatives etc. the patients were treated with topical medical ointments to relax the anal sphincter and were advised to apply it at lest 4 times per day and seen regularly every 2 weeks. 207 patients healed after 2 weeks (36.5\%), 147 after 1 month (26\%), $90(16 \%)$ between $4-12$ weeks and $72(12.7 \%)$ patients needed treatment for longer than 12 weeks. 10 patients were treated by botox and registered complete healing and 25 patients $(4.4 \%)$ required an internal sphincterotomy for complete healing that was performed in the fissure bed involving only the sphincter up to the apex of the fissure.

We have noticed a direct relationship between the duration of fissure symptoms and the period of healing.

The most important thing during the period of treatment is good communication with the patient, detailed explanations of different methods of treatment, advantages and disadvantages, risk of side-effects, incontinence and adapt to the symptoms and psychology of each patient. The patient should be instructed to continue performing sitz baths, taking fibers, drinking plenty liquids, using laxatives, glycerin suppositories, small enemas to unblock the stools, and apply rigorously and very correctly the topical treatment. Generally, even in patients with the worst symptoms there is a clear consistent improvement after 5-6 days in intensity and duration of pain. The patient should be given as an orientation for the progression of treatment to follow those 2 aspects and report them at each visit. Also video documentation should be used to monitor healing and motivate the patient (Figs. 1 and 2).

Even after the healing the patient should remain on a rich fiber diet, with plenty of liquids, avoid constipation by glycerin suppositories, small enemas or soft laxatives to avoid hard stools. In our experience, constipation was the main cause for the recurrence of the fissure. The medical

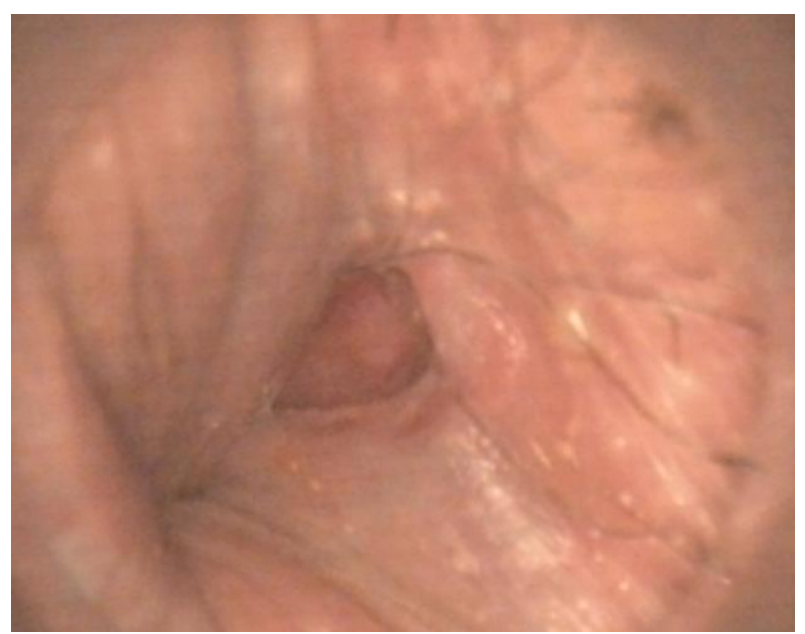

Fig. 1 Anterior CAF with clean edges, some granulation tissue visible in the fissure bed, triangular shape aspect in evolution under medical treatment

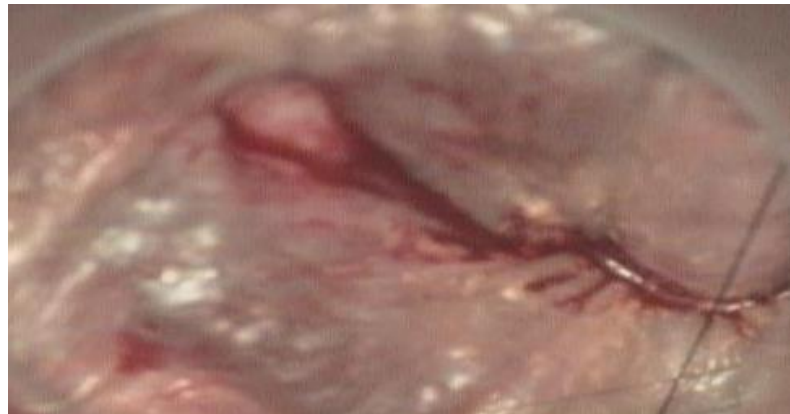

Fig. 2 Posterior chronic anal fissure with fibroses in the fissure's bed, granulation tissue on the edges, under medical treatment

treatments as NTG, CCB are useful and cheaper but necessitate administration in at least two times/daily, best 4-5 times for a period of time from 2 weeks to 12 weeks, the median is 6-8 weeks. We noticed a linear relationship between the period of anal fissure and the time of healing, the longer the history of fissure, more time is taking to complete healing. The disadvantage is the necessity of multiple applications, necessitating some minimal conditions of privacy and hygiene during the day, or in the working place, some minor degree of tachyphylaxis. The patient should be seen every two weeks to be sure that the applications is correct, the diet is correct and adjust treatments by changing topical treatments

Botox is a very good option and could be recommended after 6-8 weeks of topic treatment. The disadvantage is the cost. Otherwise, the botox therapy could be very well proposed to the patients from the first place. In patients that are not compliant from any reason for topical treatment this could be recommended from the first place

The choice between medical treatments of botox, the sequence, the decision to inject botox should always comply with the patient. Remember that we treat patients and not disease. Detailed discussion with the patient, good communication, details about habits, diet, style of life, job at least should be obtained, the advantage $s$ and disadvantages of every type of treatment should be exposed and the decision taken after considering at least all those factors and the choice of patient.

In conclusion, the conservative, non-operative treatment should be pushed as much as possible, and surgery avoided as much as possible, as long as the pain and compliance of the patient otherwise passage to surgery is the most reliable and sure thing to heal.

The conservative treatment involves a great deal of work and communication with the patient, but it may be rewarding at the end, and even if a recurrence take place this is due to mainly to patient lack of adherence to the principles of diet. Of course, each recurrence may be treated with success again by medical non-surgical therapy and again the decision should be taken together with the patient and if necessary surgery will be performed when compliance of the patient is obtained.

Acknowledgment: The authors have no conflicts of interest. 


\section{References}

1. Espin E. Anal fissure. In: Herold A, Lehur P-A, Matzel KE, O’Connel PR, eds. Manual of coloproctology. 2nd edition. Berlin Heidelberg: Springer Verlag; 2017. p. 47-59.

2. Lund JN, Scholefield JH. Aetiology and treatment of anal fissure. Br J Surg 1996; 83:1335-1344.

3. Schouten WR, Briel JW, et al. Relationship between anal pressure and anodermal blood flow: the vascular pathogenesis of anal fissure. Dis Colon Rectum 1994; 37:664-669.

4. Gibbons CP, Read NW. Anal hypertonia in fissures: Cause or effect? Br J Surg 1986; 58:96.

5. Kodner IJ, Fry RD, Fleshman J W, Birnbaum EH, Read TE. Colon, Rectum and Anus. In: Seymour I. Schwartz et al, eds. Principles of Surgery. 7th ed. Mc Grow-Hill; 1999. p.1265-1382.

6. Jenkins JT, Urie A, Molloy RG. Anterior anal fissures areassociated with occult sphincter injury and abnormalsphincter function. Colorectal Dis 2008; 10(3):280-285.

7. Zaghyian KN, Fleschner P. Anal fissure. Clin Colon Rectal Surg $2011 ; 24: 22-30$

8. Klosterhalfen B, Vogel P, et al. Typography of the inferior rectal artery: a possible cause of chronic primary fissure. Dis Colon Rectum 1989; 32:43-52.

9. Maria G, Brissinda D, et al. Identification of anti-endothelial cell antibodies (EACA) in patients with chronic anal fissures. Surgery 1999; 126:535-540.

10. Nelson R. Non surgical therapy for anal fissure. Cochrane Database Syst Rev 2006;(4):CD003431.

11. Nelson R. Operative procedures for fissure in ano. Cochrane Database Syst Rev 2005;(2):CD002199.

12. Jameson JS, ChiaYW, Kamm MA, Speakman CT, ChyeYH, Henry MM. Effect of age, sex and parity on anorectal function. Br J Surg 1994; 81:1689-1692.

13. Jonas M, Neal KR, Abercrombie JF, Scholefield JH. Arandomized trial of oral vs. topical diltiazem for chronic analfissures. Dis Colon Rectum 2001; 44(8):1074-1107.

14. Perrotti P, Bove A, Antropoli C, et al. Topical nifedipinewith lidocaine ointment vs. active control for treatment ofchronic anal fissure: results of a prospective, randomized,double-blind study. Dis Colon Rectum 2002; 45(11):1468-1475.
15. Nelson R. Non surgical therapy for anal fissure. Cochrane Database Syst Rev 2006; (4):CD00343

16. Scholefield JH, Bock JU, Marla B, et al. A dose findingstudy with $0.1 \%, 0.2 \%$, and $0.4 \%$ glyceryl trinitrate ointmentin patients with chronic anal fissures. Gut 2003; 52(2):264-269.

17. Lund JN, Scholefield JH. A prospective, randomized double-blind placebo controlled trial of glyceryl trinitrate in anal fissure. Lancet 1997; 349:11-14.

18. Maria G, Casetta E, et al. A comparison of botulinum toxin and saline injection for treatment of chronic anal fissure. N Engl J Med 1998; 338:217-220.

19. Bobkiewicz A, Francuzik W, Krokowicz L, Studniarek A Ledwosiński W, Paszkowski J, Drews M, Banasiewicz T. Botulinum Toxin Injection for Treatment of Chronic Anal Fissure: Is There Any Dose-Dependent Efficiency? A Meta-Analysis. World J Surg 2016; 40(12):3064-3072.

20. Lin JX, Krishna S, Su'a B, Hill AG. Dosing of Botulinum Toxin for Treatment of Chronic Anal Fissure: A Systematic Review and Meta-Analysis. Dis Colon Rectum 2016; 59(9):886-894.

21. Nelson RL, Manuel D, Gumienny C, Spencer B, Patel K, Schmitt K, Castillo D, Bravo A, Yeboah-Sampong A. A systematic review and meta-analysis of the treatment of anal fissure. Tech Coloproctol 2017; 21(8):605-625.

22. Sahebally SM, Meshkat B, Walsh SR, Beddy D. Botulinum toxin injection vs topical nitrates for chronic anal fissure: an updated systematic review and meta-analysis of randomized controlled trials. Colorectal Dis 2018; 20(1):6-15.

23. Wald A, Bharucha AE, Cosman BC, Whitehead WE. ACG clinical guideline: management of benign anorectal disorders. Am J Gastroenterol 2014; 109(8):1141-1157;

24. Beck DE, Timmcke AE. Pruritus ani and fissure-in-ano. In: Beck DE, ed. Handbook of Colorectal Surgery. 2nd ed. New York: Marcel Dekker; 2003. p. 367.

25. Lund JN, Scholefield JH. Aetiology and treatment of anal fissure. Br J Surg 1996; 83:1335-1344.

26. Sileri P, Stolfi VM, Franceschilli L. Grande M, di Giorgio A D'Ugo S, Attina G, D'Eletto M, Gaspari AL. Conservative and surgical treatment of chronic anal fissures: prospective long-term results. J Gastrointest Surg 2010; 14:773-780. 